\title{
涌现计算：从无序掌声到有序 掌声的虚拟现实
}

\author{
李德毅 ${ }^{1}$ 刘 坤 $2^{*}$ 孙 岩 ${ }^{1}$ 韩明畅 ${ }^{1}$ \\ (1. 中国电子系统工程研究所, 北京 100039; 2. 解放军理工大学指挥自动化学院, 南京 210007)
}

\begin{abstract}
摘要以音乐厅自发同步掌声作为研究对象, 建立了一个描述复杂多个体系统 集体行为的非线性涌现模型. 基于这个模型, 开发了一个仅仅依靠局部相互作用 的涌现计算实验平台, 揭示了涌现行为的不确定性和多样性. 通过对多人鼓掌过 程数据的分析, 发现了观众的掌声在从无序转向同步过程中存在一个明显的临界 区域，并得到了掌声同步的基本判据：若耦合系数 $c_{1}$ 和 $c_{2}$ 满足条件 $0.02 \leqslant c_{2} \leqslant$ $0.965 c_{1}+0.018$, 则掌声能够实现同步.
\end{abstract}

\section{关键词涌现计算 同步 从众心理 耦合函数}

\section{1 引言}

从核子到宇宙、从生态到人类社会、从物理到化学, 在不同尺度上、不同领域里, 都广泛 存在着自发涌现的集体同步现象, 如萤火虫一致发光, 一群长期居住在一起的妇女月经周期 趋向相同, 音乐厅里人群自发鼓掌同步, 蟋蟀群的叫声有节奏地唱和, 神经元的同步放电斑图, Huygens摆同步现象等. 涌现是复杂系统内部个体之间通过局部相互作用, 产生在系统规模上 才能观察到的一些新属性和新现象 ${ }^{[1]}$. 这种从一种状态突变到另一种状态的现象, 在物理学中 称之为相变. 涌现研究整体大于局部之和的机理 ${ }^{[2]}$. 每个人都曾有过这样的生活经历, 在一个 精彩的节目表演结束后, 观众会爆发出雷鸣般的掌声, 在很短的时间内这种嘈杂的掌声会突 然转变成有节奏的掌声, 似乎有一股神秘的力量驱使观众一致地鼓掌, 这是一个典型的复杂 系统自组织同步现象(下文称为“掌声响起来”).

关于同步问题的研究有着非常悠久的历史, Wiener, Winfree, Kuramoto和Strogatz等著名的 科学家在这一领域都做出过突出的贡献, 如Winfree模型和Kuramoto模型的提出. 这些模型的 一个共同点就是假定系统中每个个体具有相同的振动周期, 然后将同步问题转化为相同步问 题 ${ }^{[3,4]}$. 随着计算机计算能力的增强, 人们开始用计算机模拟来研究复杂系统的各种涌现现象.

收稿日期: 2006-11-01; 接受日期: 2007-08-03

国家重点基础研究发展计划(批准号: 2007CB310800)、国家自然科学基金(批准号: 60375016, 60496323, 60675032)资助项目

* 联系人, E-mail: lmil2003@163.com 
1987 年, Reynolds构造了一个数学模型来模仿鸟群和鱼群的运动, 简称为Boid模型 [5]. 在Boid 模型中, 每个个体仅根据它附近个体的行为调整自己的行为, 在运动过程中遵循避免碰撞、速 度一致和向中心聚集 3 条基本规则. 1995 年, Vicsek等提出一个简单的数学模型来研究非平衡 系统中的聚类、运送和相变. 该模型考虑有限个自主运动的个体(粒子、点和动物等), 并假定 每个个体以恒定的速率运动, 在给定时刻, 每个个体的运动方向为上一时刻它的邻居运动方 向的平均 ${ }^{[6]}$. 事实上, Vicsek模型只考虑了Boid模型中的速度匹配规则, 因此, 从某种意义上说 它是Boid模型的一个特例.

2000 年, Néda等通过分析音乐厅里录制的真实掌声, 得出掌声同步时周期加倍的结论 ${ }^{[7]}$, 并借助Kuramoto模型, 对这一结论给出了一个理论解释 ${ }^{[8]}$. Kuramoto模型是一个典型的相同步 模型, 它并不反映振子之间周期的相互作用. Néda等在实验中通过人为干预使振子振动周期加 倍, 验证了其研究结论, 但他们的研究具有一定的局限性, 且未能实现真正意义上的自发同步 涌现，也未能揭示实际生活中观众鼓掌行为的多样性.

本文选取 “掌声响起来”作为研究对象，基于人类心理学中普遍存在的“从众心理”[9,10]，简 化并明确 “掌声响起来” 的初始状态, 利用认知物理学中的云模型和数据场理论 ${ }^{[11]}$, 构造了一 个描述集体行为的非线性涌现模型. 基于这个模型, 本文开发了仅依靠局部相互作用而不存 在统一调度的涌现计算实验平台, 仔细分析了音乐厅里掌声的自发涌现机理, 并用计算机模 拟了各种各样的虚拟现实.

\section{2 “掌声响起来”的初始状态}

为了深入研究涌现的机理, 我们首先对 “掌声响起来”问题做了必要的形式简化.

在研究大量个体之间的相互作用而形成的涌现现象时, 常常假定单个个体的行为非常简 单. 对于音乐厅多人掌声的研究, 集中在每个观众的鼓掌行为上, 他们在性别、年龄和心情等 方面的差异可以忽略. 无论是混乱的掌声还是同步的掌声, 每个观众的鼓掌行为实际上都不 是一个单一频率, 也不是一个周期信号, 我们不妨用起拍时间 $\left(t_{0}\right)$ 、击掌间隔时间 $\left(\Delta t_{\mathrm{i}}\right)$ 和掌声强 度 $(Q)$ 描述个体行为, 它们大致对应单一频率波形的初始相位、周期和振幅. 每一个观众当前 的击掌时刻( $t_{i}$ 等于他(她)的起拍时间与到此前为止所有击掌间隔时间的总和，如图 1 所示.

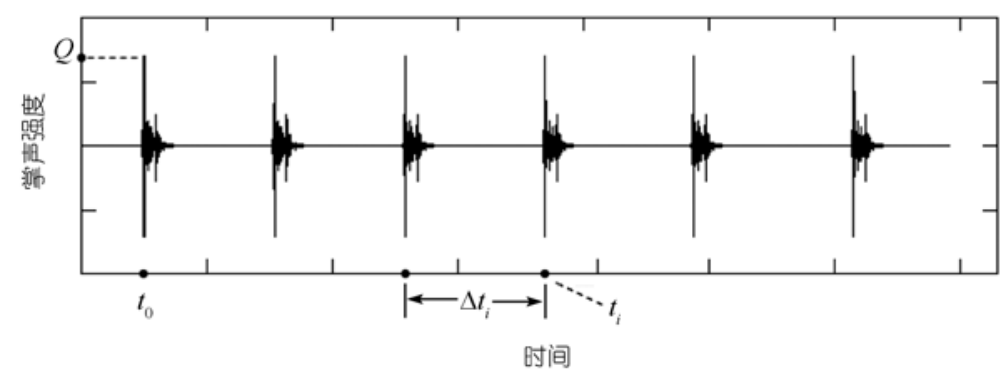

图 1 鼓掌行为的参数化表示

根据中心极限定理, 大量的、独立的单个微小事件组合成的整体行为, 常常表现出正态分

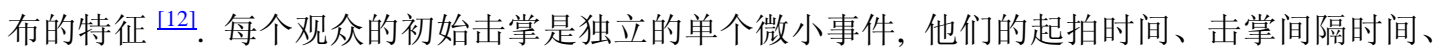
掌声强度和鼓掌次数的初始值可以近似认为服从泛正态分布. 云是用语言值描述的某个定性 概念与其数值表示之间的不确定性转换模型 [13], 它通过 2 阶正态分布方法形成泛正态分布, 
主要反映自然界中两种不确定性: 模糊性(边界的亦此亦彼性)和随机性(发生的概率). 云模型 用期望、熵和超熵 3 个数字特征来表征一个概念, 这是定性概念的整体定量描述, 可以通过这 3 个数字特征获得指定数量的云滴, 每个云滴都是对定性概念的一次随机实现. 图 2 是通过云 模型产生的 1000 个观众的击掌间隔时间初始状态的分布. 云模型的普适性也已经被证明 [14]. 因此，每个观众的起拍时间、击掌间隔时间、掌声强度和鼓掌次数的初始值可以用云模型来生 成. 用云模型产生的初始状态, 充分反映了 “掌声响起来”的不确定性, 由此形成的从无序到有 序的过程，每次都是不同的.

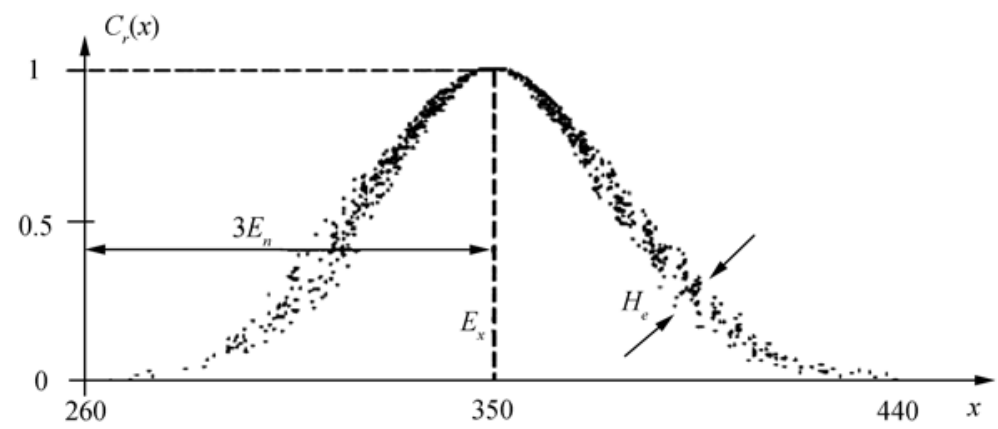

图 2 云模型及其 3 个数字特征 $\left(E_{x}=350, E_{n}=30, H_{e}=0.5\right)$

也许, 小的音乐厅和大的露天音乐会有明显的不同, 后者有声音传播速度带来时延的影 响. 至于各种音乐厅在大小、结构和环境上带来的音响效果的差异, 只要参与的观众足够多, 这些差异可以被忽略.

\section{3 “掌声响起来”的相互作用}

观众之间由于相互作用而最终实现了掌声同步. 对一个好节目的欣赏, 从社会心理学的 角度来看，与观众的互动密切相关. 由于观众的 “从众心理”, 气氛相互感染，在鼓掌过程中， 每个观众根据周围人发出掌声的快慢和早晚来调节自己的击掌节奏. 反映在观众的鼓掌行为 上，就是观众根据周围人的击掌间隔时间和击掌时刻去自发地调节他(她)的下一次击掌间隔 时间，形成一定程度的正反馈 ${ }^{[15]}$. 观众之间的距离越近，相互影响越大; 观众的掌声强度越大， 对周围人的影响越强. 每个观众受到周围其他观众的影响, 同时这个观众又作为周围人去影 响其他观众, 不存在统一的指挥. 掌声在传递过程中强度会随着距离的增加而衰减.

我们采用认知物理学中数据场理论 ${ }^{[11]}$ 来研究观众之间的相互作用, 设在有 $M$ 行和 $N$ 列座 位的音乐厅里, 观众组成 $M \times N$ 的矩阵, 每个观众的鼓掌被看作一个声源, 处在矩阵中 $(i, j)$ 位 置上观众 $A_{i, j}$ 发出的掌声传播到 $(x, y)$ 位置上的势为

$$
\varphi_{i, j}(x, y)=Q_{i, j} \times \mathrm{e}^{-\left((x-i)^{2}+(y-j)^{2}\right) / \sigma^{2}},
$$

其中 $Q_{i, j}$ 为观众 $A_{i, j}$ 的掌声强度, $\sigma$ 为距离影响因子, 它与观众 $A_{i, j}$ 的影响半径 $r$ 的关系为 $\sigma=(\sqrt{2} r / 3)$, 其中 $1 \leqslant x \leqslant M, 1 \leqslant y \leqslant N, 1 \leqslant i \leqslant M, 1 \leqslant j \leqslant N$.

定义 $\Gamma_{x, y}$ 为 $A_{x, y}$ 周围观众组成的集合, 即

$$
\Gamma_{x, y}=\left\{A_{i, j} \mid 0<\sqrt{(x-i)^{2}+(y-j)^{2}} \leqslant r\right\},
$$


集合 $\Gamma_{x, y}$ 中的每个观众的掌声都会对观众 $A_{x, y}$ 产生影响, 这些观众在 $(x, y)$ 位置上的势可以通 过叠加求得

$$
\phi(x, y)=\sum_{A_{i, j} \in \Gamma_{x, y}}\left(Q_{i, j} \times \mathrm{e}^{-\left((x-i)^{2}+(y-j)^{2}\right) / \sigma^{2}}\right) .
$$

观众 $A_{x, y}$ 的下一次击掌间隔时间 $\Delta t_{x, y}^{\prime}$, 是由他(她)自身的上一次击掌间隔时间 $\Delta t_{x, y}$ 以及 周围观众 $A_{i, j}\left(A_{i, j} \in \Gamma_{x, y}\right)$ 的最近一次的鼓掌行为(由鼓掌的快慢和早晚两个部分构成)决定的, 形式化描述为

$$
\begin{aligned}
\Delta t_{x, y}^{\prime}= & \Delta t_{x, y} \\
+ & \frac{\sum_{A_{i, j} \in \Gamma_{x, y}}\left\{\left[c_{1}(t)\left(\Delta t_{i, j}-\Delta t_{x, y}\right)+c_{2}(t)\left(t_{i, j}-t_{x, y}\right)\right] \times Q_{i, j} \times \mathrm{e}^{-\left((x-i)^{2}+(y-j)^{2}\right) / \sigma^{2}}\right\}}{\sum_{A_{i, j} \in \Gamma_{x, y}} Q_{i, j} \times \mathrm{e}^{-\left((x-i)^{2}+(y-j)^{2}\right) / \sigma^{2}}},
\end{aligned}
$$

其中 $c_{1}(t)$ 和 $c_{2}(t)$ 为耦合函数, $c_{1}(t)$ 反映 $A_{x, y}$ 周围观众的击掌间隔时间对 $A_{x, y}$ 的影响强弱, $c_{2}(t)$ 反映 $A_{x, y}$ 周围观众的击掌时刻对 $A_{x, y}$ 的影响强弱; $\Delta t_{i, j}$ 和 $\Delta t_{x, y}$ 分别为观众 $A_{i, j}$ 和 $A_{x, y}$ 上 一次击掌间隔时间, $t_{i, j}$ 和 $t_{x, y}$ 分别为他们上一次的击掌时刻; 分母是所有周围人的掌声在该 点形成的势, 用以归一化. 公式(3)考虑了单个观众击掌间隔时间的差异性, 兼顾了击掌间隔 时间、击掌时刻和掌声强度等耦合因素, 能更精确地刻画掌声自发同步过程.

观众的鼓掌次数会受到现场气氛感染因子的调节，鼓掌次数太少不会形成同步，观众也 不可能一直持续鼓掌. 一般情形下, 所有观众鼓掌次数的初始值也服从泛正态分布, 在此基础 上，单个观众的鼓掌次数受到气氛感染因子的调节，可以形式化描述为

$$
L_{x, y}(t+1)=L_{x, y}(t)+c_{3}(t) \times L_{x, y}(t)=L_{x, y}(t)+K(D(t-1)-D(t)) \times L_{x, y}(t),
$$

其中, $L_{x, y}(t)$ 和 $L_{x, y}(t+1)$ 分别为观众 $A_{x, y}$ 受气氛感染因子调节前和调节后的鼓掌次数; $c_{3}(t)=K(D(t-1)-D(t))$ 为气氛感染因子, 随时间而变化; $D(t-1)$ 和 $D(t)$ 分别为 $t-1$ 和 $t$ 时刻 所有观众击掌时刻的标准差; $K$ 为标准化系数. 当现场气氛不再升温时, 所有观众的鼓掌次数 不再增加.

耦合函数 $c_{1}(t)$ 和 $c_{2}(t)$ 是时间的函数，反映观众之间的相互作用强弱随时间而变化. 特别 地, 当 $c_{1}(t)$ 和 $c_{2}(t)$ 很小乃至为 0 时, 意味着观众之间几乎不发生相互作用, 这样形成的是礼 貌性掌声, 这种掌声多发生在场景需要或礼节性的场合. 在研究涌现过程中的规律时, 很多情 形下可以用常量的耦合函数来简化问题的分析, 这时耦合函数就变成了耦合系数.

\section{4 “掌声响起来”的实验平台}

根据 “掌声响起来”的初始状态和观众间的相互作用, 以掌声作为表现手段, 建立涌现计 算的实验平台.

首先要指出的是, 掌声仅仅是一个载体形式, 用数字音频工具录制的单人单掌声可以很 容易地载入到由每个人的起拍时间及一个个击掌间隔时间形成的流数据中去, 形成单人连续 掌声, 然后通过多个单人连续掌声的混音形成音乐厅里的多人掌声. 因此, 重要的是要得到每 个人在相互作用影响下的击掌间隔时间形成的流数据. 
为说明虚拟实验平台的流数据的生成方法, 设想一个 $M=N=8$ 的掌声矩阵, 观众鼓掌的 初始状态用云模型生成, 观众之间的影响半径 $r=3$. 鼓掌时, 根据当前每个观众的击掌时刻找 出当前最先鼓掌的观众, 按照公式(3)计算出他(她)的下一次击掌间隔时间. 接下来, 再根据击 掌时刻寻找下一个最先鼓掌的观众, 重复这一计算过程, 直至鼓掌完毕. 如图 3 所示, 图中左 边是抽取 $8 \times 8$ 方阵中处在 $(4,5)$ 位置及其周围位置上的 13 个观众的击掌间隔时间流数据, 详细 说明流数据的生成过程, 图中每行代表的那个观众自起拍至结束的全部击掌间隔时间皆用数 字标出, 单位为 $\mathrm{ms}$, 小圆圈的横轴位置表示实际的击掌时刻. 以 $A_{4,5}$ 为例, 第 1 个数据 249 表 示他(她)的起拍时间为 $249 \mathrm{~ms}$, 后面是他(她)的随后两次击掌间隔时间 $345 \mathrm{~ms}, 363 \mathrm{~ms}$, 小圆圈 中的数字是他(她)在这 13 个观众全部鼓掌中的顺序, 即 $A_{4,5}$ 的第 $1,2,3$ 次鼓掌分别是 13 个观 众中的第 2, 14, 27 次鼓掌. 当所有观众击掌时刻足够接近时，掌声同步听起来已十分明显.

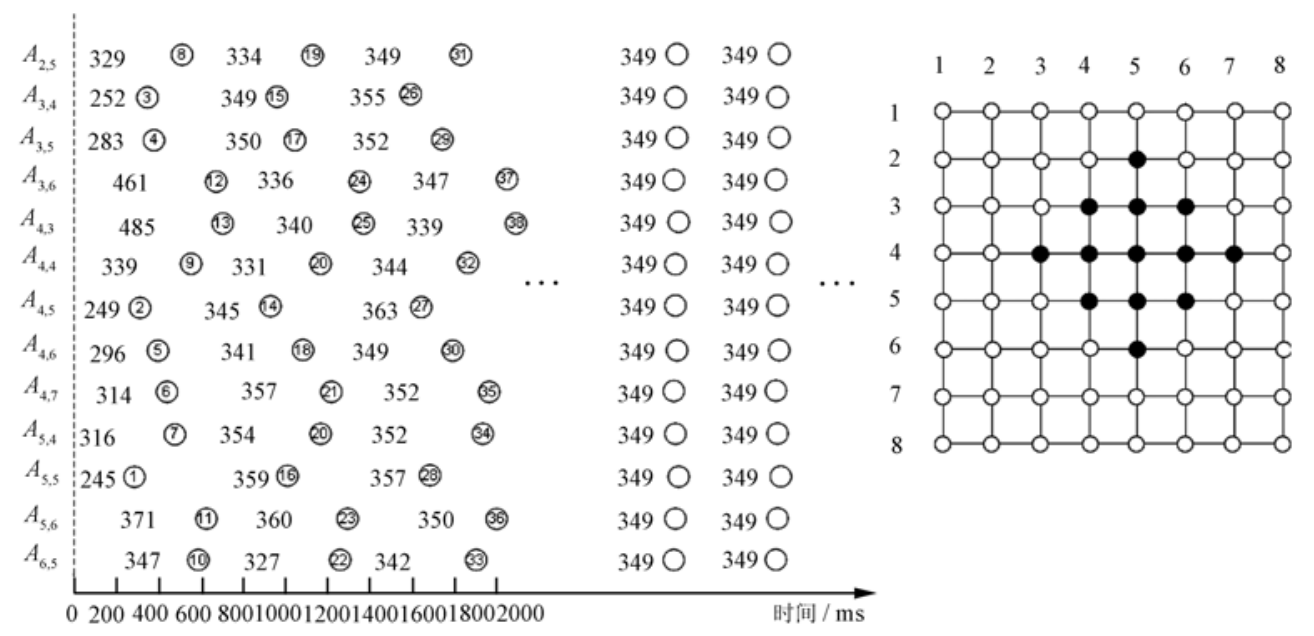

图 3 数据流文件的生成

公式(3)和流数据的生成过程表明, 每个观众只受周围影响半径 $r$ 范围内的其他观众的影 响, 每个观众只能利用他(她)周围的局部信息来调节自己, 整个过程中不存在一个全局的控 制，没有一双看不见的“手”对观众进行统一的指挥. 按照此算法, 很容易将这种过程扩展更大 规模的矩阵中去, 如 $16 \times 16$ 或 $32 \times 32$ 的观众规模.

实验计算记录了音乐厅里每个观众一次次击掌间隔时间的流数据, 通过它们可计算出相 应的击掌时刻, 然后将录制的单人单掌声载入到流数据文件中的这些时刻点上, 形成单人连 续掌声文件, 再用多媒体混音算法 [16]对所有观众的单人连续掌声文件进行合成, 得到多人鼓 掌的声音文件, 通过数字音频播放器(如Windows Media Player)播放.

通过实验平台(交互界面如图 4 所示), 可以设定观众规模和鼓掌初始状态; 进一步选择音 乐厅、大操场等不同的耦合模式, 调节耦合函数, 便可以生成不同类型的掌声涌现效果. 平台 下半部分的单人掌声矩阵用以形成局部观众的连续掌声文件, 可任意选择组合区域, 得到该 区域群体的合成掌声.

由于考虑了随机性, 实验平台每一次生成的掌声都不会相同. 尽管如此, 人们普遍认为它 与在音乐厅中录制的真实掌声在声音效果上很难区分, 图 5 给出典型的例子. 我们让几个学生 分别试听这些不同的声音, 根本区分不出哪个是录制的真实掌声, 哪个是合成的掌声, 也没有 
两次完全相同的掌声, 充分体现了实验计算的不确定性. 通过改变实验平台初始参数, 如让耦 合系数 $c_{1}=c_{2}=0$, 可以得到礼貌性掌声, 也可以改变 $c_{1}$ 和 $c_{2}$ 的值, 得到从混乱到有序的各种 自发同步掌声，甚至可以出现 2 次同步或间歇同步的情形，表现出涌现的多样性.

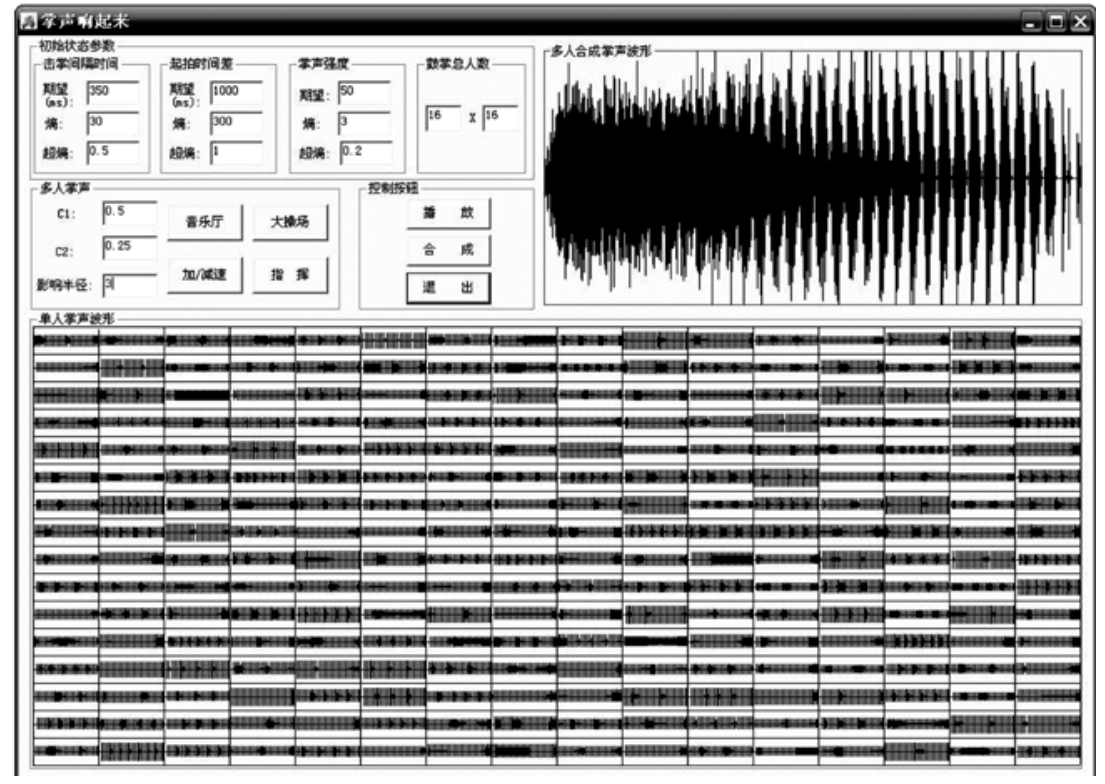

图 4 掌声响起来实验计算平台
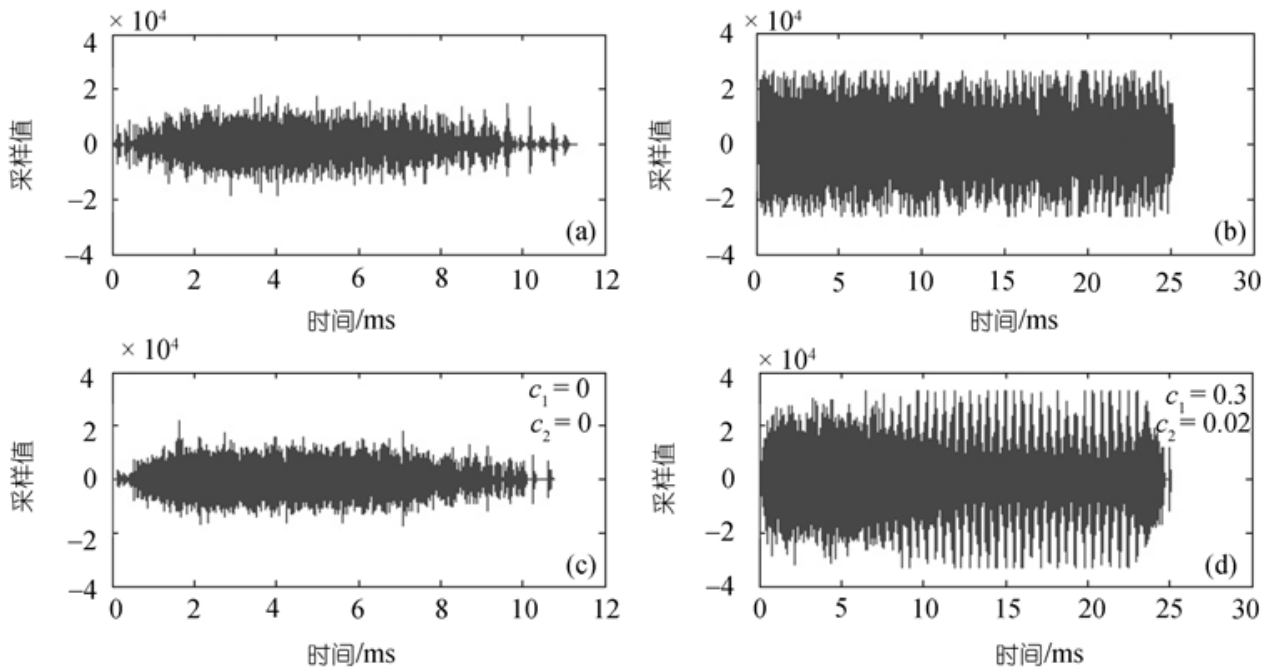

图 5 虚拟掌声与真实掌声的比较

(a) 录制的礼貌性掌声; (b) 录制的自发同步掌声; (c) 虚拟的礼貌性掌声; (d) 虚拟的自发同步掌声

\section{5 实验数据分析及涌现的一般规律}

从虚拟掌声的生成过程可知, 单人单掌声仅仅是合成掌声数据流文件中的一系列击掌时 刻事件的载体形式, 以满足实验计算的多媒体表现需要. 因此, 为了分析涌现的一般规律, 我 
们可以将掌声这种载体形式剥离出去, 直接对实验计算记录的流数据进行分析.

\section{1 从无序到有序的过程分析}

观众刚开始的鼓掌是混乱的, 由于相互作用, 同步掌声突然涌现出来, 涌现过程必然存在 着从混乱到有序的临界区. 熵是描述热力学系统混乱程度的经典方法 ${ }^{[17]}$, 我们借用熵理论来 分析多人鼓掌过程中的混乱程度.

设音乐厅里的总人数为 $m$, 在 $\Delta t$ 时间窗口内, 将观众划分为击掌和不击掌两种状态, 统 计窗口内击掌的观众数为 $n$, 则鼓掌的概率为 $P(A)=n / m$, 不鼓掌的概率为 $P(\bar{A})=(m-n) / m$. 根据 Shannon 熵的定义, 在 $\Delta t$ 时间内的音乐厅里观众鼓掌的混乱程度可以表示为

$$
H=-[P(A) \times \log P(A)+P(\bar{A}) \times \log P(\bar{A})] .
$$

若所有观众同时击掌或不击掌, 即 $P(A)=1$ 或 $P(A)=0$ 时, 此时熵 $H=0$, 掌声同步; 如果 恰好有一半人击掌, 即 $P(A)=P(\bar{A})=0.5$ 时, 熵 $H$ 达到极大值 0.7 , 掌声最无序. 因此, 熵 $H$ 的 变化可以反映多人鼓掌时的混乱和有序状况, 当熵 $H=0$ 或足够小时, 掌声达到同步.

时间窗口的宽度对熵值 $H$ 的变化有一定的影响. 窗口太宽, 会使同一个观众的连续击掌 都落入同一个窗口, 熵值精度不够; 窗口太窄, 当听起来掌声明显同步时, 多人击掌时刻的细 微偏差仍然会反映到熵值上. 根据从混乱到同步的流数据分布, 特别是达到同步状态时的击 掌时刻的分布宽度(参见图 3), 我们微调窗口的宽度, 使熵 $H$ 的变化可以反映音乐厅掌声的混 乱程度, 确定窗口的最佳宽度为 $250 \mathrm{~ms}$.

分别选取音乐厅里的一段礼貌性掌声和一段自发同步掌声, 用熵进行对比分析, 如图 6(a) 所示. 礼貌性鼓掌时, 观众鼓掌行为仅为自身状态, 彼此间几乎无耦合作用. 流数据分析显示, 这种鼓掌开始后会迅速变乱, 持续一段很短的时间后迅速结束. 相反, 在自发同步情形下, 由 于节目精彩, 从众心理使得观众之间相互耦合, 开始的混乱逐步减弱, 有序性涌现出来. 当熵 曲线达到 0 时, 形成同步的掌声; 当熵曲线结束时, 鼓掌过程结束. 自发同步掌声在从混乱转 向有序时, 存在一个明显的临界区(从 $6.3 \mathrm{~s}$ 到 $10.85 \mathrm{~s}$ ), 如图 6(b)所示.

\section{2 掌声同步涌现的判据}

耦合系数 $c_{1}$ 反应观众之间击掌间隔时间的影响程度, 耦合系数 $c_{2}$ 反应观众之间击掌时刻 的影响程度, $c_{1}$ 与 $c_{2}$ 的不同组合反映音乐厅里观众之间相互作用的强弱和主导因素, 不同的组 合会产生不同的鼓掌结果. 有的组合可以形成同步, 有的组合则不能形成同步, 有的组合看似 要形成同步但却又没能形成同步. 在能形成同步的 $c_{1}$ 和 $c_{2}$ 组合中, 其达到同步的速度也是不 一样的.

以 $16 \times 16$ 规模的中小型音乐厅为研究对象, 假设观众的上座率为 $100 \%$, 用云模型生成每 个观众的初始状态. 在公式(3)中, 均匀选择 $c_{1}$ 和 $c_{2}$ 的样本点, 统计每种组合是否能够形成同步 的掌声, 并通过拟合边界曲线得到结果如图 7 所示. 从图 7 中可以发现, 在由 $c_{1}$ 和 $c_{2}$ 组成的平 面上 $\left(0 \leqslant c_{1} \leqslant 1,0 \leqslant c_{2} \leqslant 1\right)$, 直线 $L_{1}: c_{2}=0.965 c_{1}+0.018$ 和 $L_{2}: c_{2}=0.02$ 把平面分成同步与不同 步两个区域. 直线 $L_{1}$ 和 $L_{2}$ 以及直线 $c_{1}=1$ 构成一个三角形区域, 在这个三角形区域以外的 $c_{1}$ 和 $c_{2}$ 组合不能形成同步的掌声, 而在三角形区域内的 $c_{1}$ 和 $c_{2}$ 组合能够形成同步的掌声. 进一步的 分析可以发现, 在三角形区域内, 所有 $c_{1}$ 和 $c_{2}$ 的组合始终有 $c_{1}>c_{2}>0$, 这说明形成自发同步要 
求击掌间隔时间耦合要比击掌时刻耦合更强, 且击掌时刻耦合不能太弱. 因此, 基于我们的模 型, 可以给出一个形成掌声同步的判据: 即当耦合系数 $c_{1}$ 和 $c_{2}$ 满足条件 $0.02 \leqslant c_{2} \leqslant 0.965 c_{1}+$ 0.018 时，模型表示的音乐厅自发掌声能够形成同步.
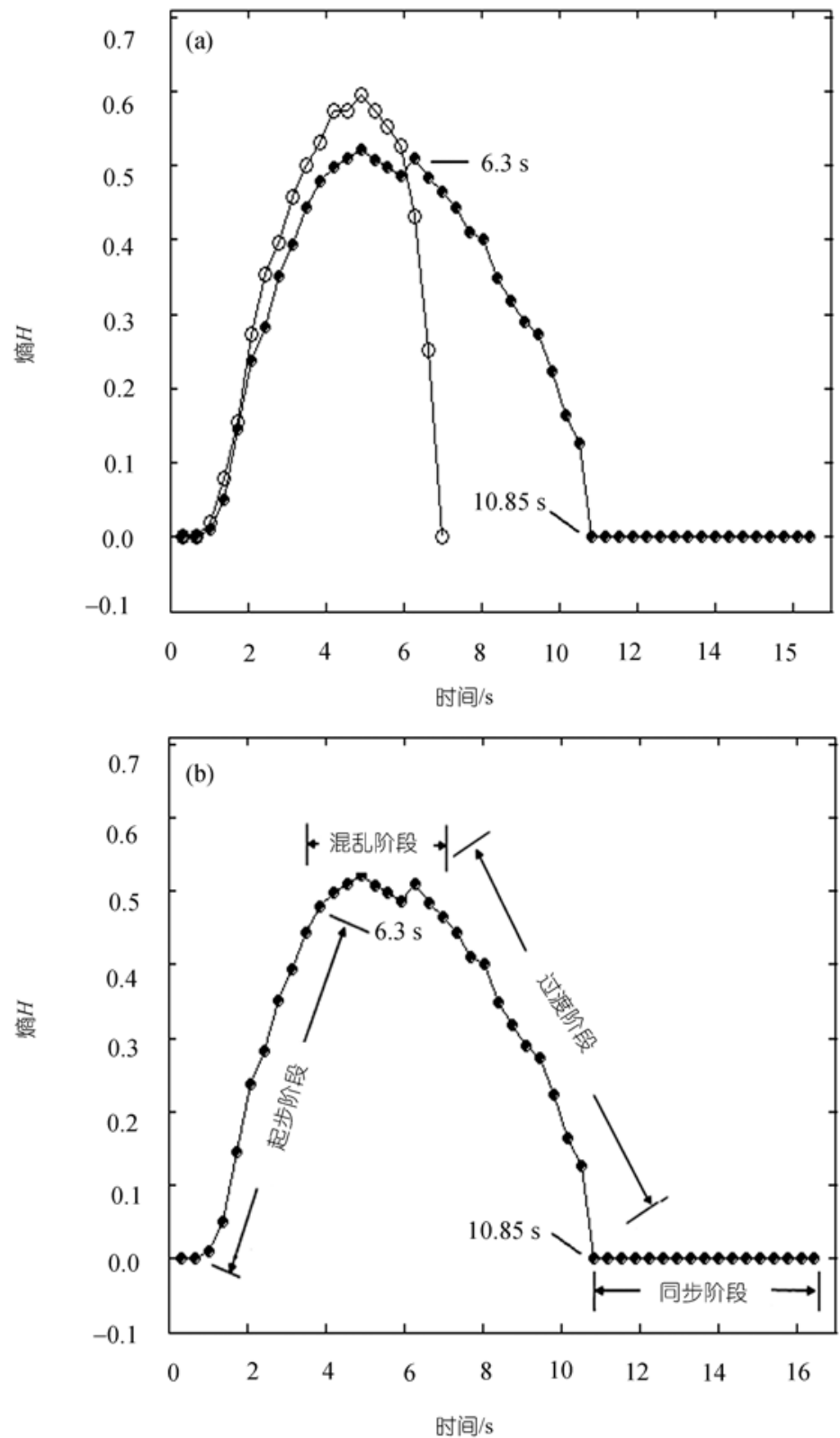

图 6

(a)音乐厅礼貌性掌声与自发同步掌声的熵曲线对比： $O$ 礼貌性掌声 $\left(c_{1}=0, c_{2}=0, E(\Delta t)=350 \mathrm{~ms}\right)$,

音乐厅掌声 $\left(c_{1}=0.4, c_{2}=0.05\right.$, $E(\Delta t)=350 \mathrm{~ms})$; (b)音乐厅自发同步掌声从混乱到有序的变化过程 $\left(c_{1}=0.4, c_{2}=0.05, E(\Delta t)=350 \mathrm{~ms}\right)$

\section{6 今后工作}

涌现是复杂系统的共性, 由于个体间相互作用的多样性, 涌现往往表现出很多具体的形 式. 为此, 我们将进一步考虑同步机理的更多因素, 对掌声同步现象进行更深入的研究. 


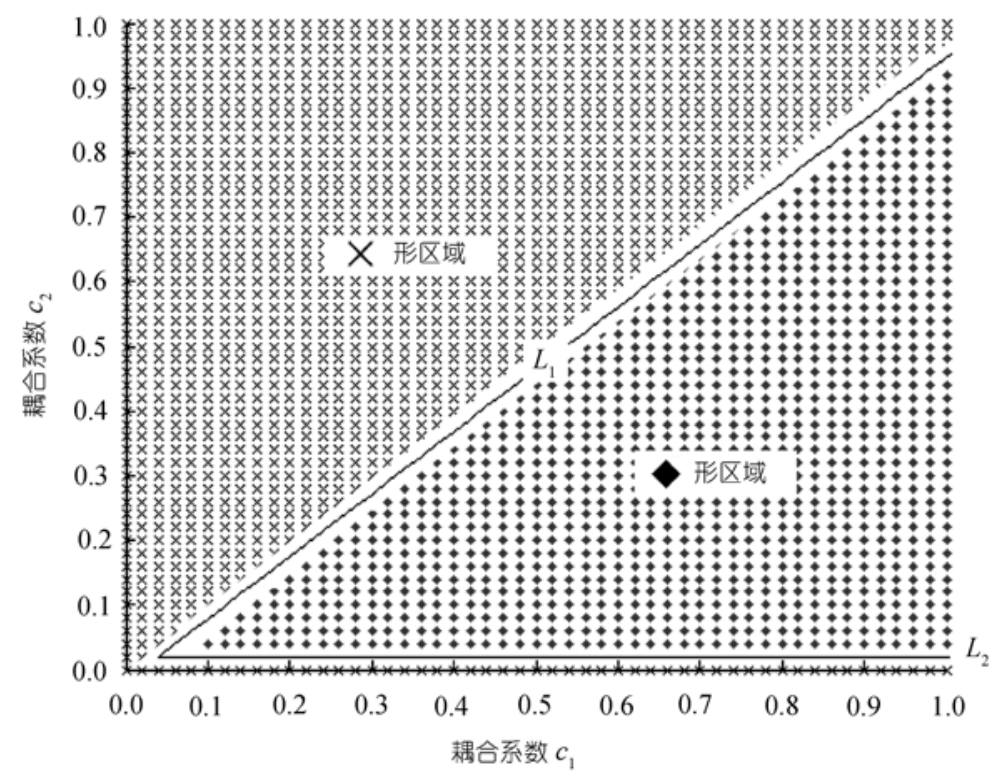

图 7 由 $c_{1}$ 和 $c_{2}$ 组成的平面上的同步区域划分

$\times$ 表示不能形成同步的区域, $\bullet$ 表示容易形成同步的区域; $L_{1}: c_{2}=0.965 c_{1}+0.018, L_{2}: c_{2}=0.02$

骨干带动下的同步：音乐厅表演时，常有若干观众按照预先准备带头鼓掌，以提升场内气 氛. 这些观众的鼓掌往往不受其他观众的影响, 而是以隐蔽的方式引导场内自发高潮的来临, 对整体气氛起带动作用. 当气氛趋向热烈时，这种带动对周围人形成正反馈，形成同步掌声. 反之, 当观众热情消退时, 他们的带动可以维持整体气氛, 延长同步时间, 甚至重新激发起整 体热情, 使鼓掌进入下一个同步过程. 在实验中, 明显观察到这种同步比自发同步要迅速而明 显.

2 次同步: 耦合系数 $c_{1}$ 和 $c_{3}$ 反映周围人对自身的影响程度, 与观众的热情程度相关, 可以 设为随时间变化的函数 $c_{1}(t)$ 和 $c_{2}(t)$. 观众在观看一场演出的氛围, 不可能一直都保持很高的热 情, 耦合强度会从高峰衰减, 伴随观众热情的回升又逐步增加. 这一形势, 反映为混乱转变为 同步，同步后又混乱，而后再同步的 2 次同步现象，我们对此已经做了实验研究，可以虚拟再 现.

露天音乐会掌声的涌现: 涌现具有多样性, 音乐厅里出现的同步掌声只是涌现的一种形 式. 如果是露天音乐会, 广场空间会很大, 声音传播的时延问题变得明显. 考虑到标准音速为 $340 \mathrm{~m} / \mathrm{s}$, 对于在体育场举行的露天音乐会, 前后观众距离可能在百米以上, 听到远方鼓掌时 会有几百毫秒的延时, 加之音响效果差, 使得彼此的耦合作用微弱. 因此, 当随情节发展在局 部区域观众中产生自发同步掌声时, 难以影响远方的局部区域, 存在时间差或相位差, 形成此 起彼伏的掌声, 这一现象将是我们下一阶段研究的重点.

致谢 感谢中国科学院数学与系统科学研究院吕金虎教授对本文工作所提出的建议; 感 谢中国电子系统工程研究所信息资料研究中心刘俊平高工对本文写作给予的帮助.

\section{参考文献}

1 Holland J H. Emergence: From Chaos to Order. Redwood City: Perseus Books, 1998 
2 von Bertalanffy L. 一般系统论基础, 发展和应用. 林康义, 魏宏森, 等译. 北京: 清华大学出版社, 1987. 70一72

3 Huang D B. Synchronization-based estimation of all parameters of chaotic systems from time series. Phys Rev E, 2004, 69(6): 067201-067204 [DOI]

4 Huang D B. Identifying parameter by identical synchronization between different systems. Chaos, 2004, 14(1): 152-159 [DOI]

5 Reynolds C W. Flocks, herds, and schools: A distributed behavioral model. In: Proc ACM SIGGRAPH'87 Conf, Comput Graph, vol 21. Anaheim: ACM Press, 1987. 25-34

6 Vicsek T, Czirok A, Jacob E B, et al. Novel type of phase transition in a system of self-driven particles. Phys Rev Lett, 1995, 75: 1226-1229 [DOI]

7 Néda Z, Ravasz E, Brechet Y, et al. The sound of many hands clapping—— Tumultuous applause can transform itself into waves of synchronized clapping. Nature, 2000, 403: 849-850 [DOI]

8 Néda Z, Ravasz E, Vicsek T, et al. Physics of the rhythmic applause. Phys Rev E, 2000, 61(6): 6987-6992 [DOI]

9 Tesser A. Advanced Social Psychology. New York: McGraw-Hill Inc, 1995. 419-430

10 Zimbardo P G. The human choice: Individuation, reason, and order versus deindividuation, impulse, and chaos. In: Arnold W J, Levine D, eds. Nebraska Symposium on Motivation. Nebraska: University of Nebraska Press, 1970. 237-307

11 李德毅, 杜鹆. 不确定性人工智能. 北京：国防工业出版社, 2005. 193-212

12 王梓坤. 概率论基础及其应用. 北京: 北京师范大学出版社, 1995. 152-158

13 李德毅, 史雪梅, 孟海军. 隶属云和隶属云发生器. 计算机研究和发展, 1995, 32(6): 15-20

14 李德毅, 刘常昱. 论正态云模型的普适性. 中国工程科学, 2004, 6(8): 28-34

15 郑维敏. 正反馈. 北京: 清华大学出版社, 1998. 118-122

16 徐保民, 王秀玲. 一个改进的混音算法. 电子与信息学报, 2003, 25(12): 1709-1713

17 Shannon C E. A mathematical theory of communication. Bell Syst Tech J, 1948, 27(6,10): 379—423, 623—656 